\title{
Progress in Microtomography at the ANATOMIX Beamline of Synchrotron Soleil
}

Timm Weitkamp $^{1, *}$, Mario Scheel ${ }^{1}$, Jonathan Perrin ${ }^{1}$, Vincent Le Roux ${ }^{1}$, Victorien Joyet ${ }^{1,2}$, Samir Chaouchi $^{1}$, Hernán-Gabriel Pais ${ }^{1}$, Jean-Luc Giorgetta ${ }^{1}$, Alexandre Carcy ${ }^{1}$, Kewin Desjardins ${ }^{1}$, Claude Menneglier ${ }^{1}$, Shu Zhang ${ }^{1}$, Christer Engblom ${ }^{1}$, Matthieu Cerato ${ }^{1}$, Yves-Marie Abiven ${ }^{1}$, Gilles Cauchon ${ }^{1}$, Camille Rivard ${ }^{1,3}$, Thierry Moreno ${ }^{1}$, François Polack ${ }^{1}$ and Andrew Thompson ${ }^{1}$

1. Synchrotron SOLEIL, Gif-sur-Yvette, France.

2. Institut Laue-Langevin, Grenoble, France.

3. Department Science for Food and Bioproduct Engineering (CEPIA), French National Institute for Agricultural Research (INRA), Nantes, France.

* Corresponding author, weitkamp@synchrotron-soleil.fr

ANATOMIX is a long undulator beamline for full-field radiography and tomography with $\mathrm{X}$ rays in the energy range from 5 to $25 \mathrm{keV}$. The design principles and instrument layout have recently been reported elsewhere [1]. In brief, ANATOMIX has two experimental hutches located, respectively, 170 and $200 \mathrm{~m}$ from the X-ray source. It is located on a canted straight section of the SOLEIL storage ring, which it shares with the scanning-nanoprobe beamline NANOSCOPIUM [2]. The experimental methods at ANATOMIX are (1) absorption and phase-contrast microtomography in parallel-beam projection geometry and (2) tomography with a transmission X-ray microscope (TXM), in which a zone plate creates an enlarged real X-ray image of the sample. The status of the TXM is described in another paper in this volume [3].

ANATOMIX can take the white X-ray beam in both of its experiment hutches, but also features a double-crystal Si-111 monochromator in Bragg geometry and a double-multilayer monochromator. The beam size at sample without any optics in the beam is approximately $20 \mathrm{~mm}$ (horizontal) $\times 15 \mathrm{~mm}$ (vertical). It can be increased in the horizontal dimension to more than $40 \mathrm{~mm}$ by a meridionallyfocusing double mirror with horizontal deflection, located approximately $35 \mathrm{~m}$ from source.

Since summer 2017, the beamline has been taking white-beam microtomography data using a temporary setup. This setup consists essentially of a high-precision rotation stage (model RT150v3, LAB Motion Systems, Heverlee, Belgium) and an indirect lens-coupled detector. The rotation stage and detectors are mounted on standard translation stages (Huber Diffraktionstechnik, Rimsting, Germany) for alignment.

The indirect detectors use two different types of digital cameras: a CMOS with $2048 \times 2048$ pixels of $6.5 \mu \mathrm{m}$ physical size (model Orca Flash 4.0 V2+, Hamamatsu Photonics, Hamamatsu City, Japan) and a CCD with $4008 \times 2672$ pixels of $9.0 \mu \mathrm{m}$ size (model pco.4000, PCO, Kelheim, Germany). In the temporary setup, they are coupled to different single-crystal scintillators via two different types of optics developed in-house: a high-resolution system, based on a 10× microscope objective (Mitutoyo, Kawasaki, Japan), and a 1:1 system based on photo lenses (Hasselblad, Gothenburg, Sweden).

Figures 1 and 2 show examples of tomography data obtained with a filtered white beam, using this temporary system. The data in Figure 1 were obtained with a wide beam from the double mirror, using the 1:1 optics and the CCD-based detector, with corresponding medium spatial resolution (pixel size $9 \mu \mathrm{m})$. Figure 2 shows high-resolution data $(0.65 \mu \mathrm{m}$ pixel size $)$ acquired with the $10 \times$ optics and the CMOS camera. With the sample composed of light elements, inline phase contrast prevails here. 
The definite microtomography system, currently under commissioning, features a sample manipulator with long Z stroke $(120 \mathrm{~mm})$ and two rotation stages: a 500-mm-diameter stage with $30 \mathrm{~kg}$ load capacity and an additional smaller rotation stage for fast acquisitions. The system will enable acquisition rates of up to one tomography scan per second. Optimized detector optics systems are currently being built [4]. Where possible, the design of the experimental station aims at compatibility with the tomography station at SOLEIL's PSICHÉ beamline [5] in terms of mechanics, electronics, controls and user experience.

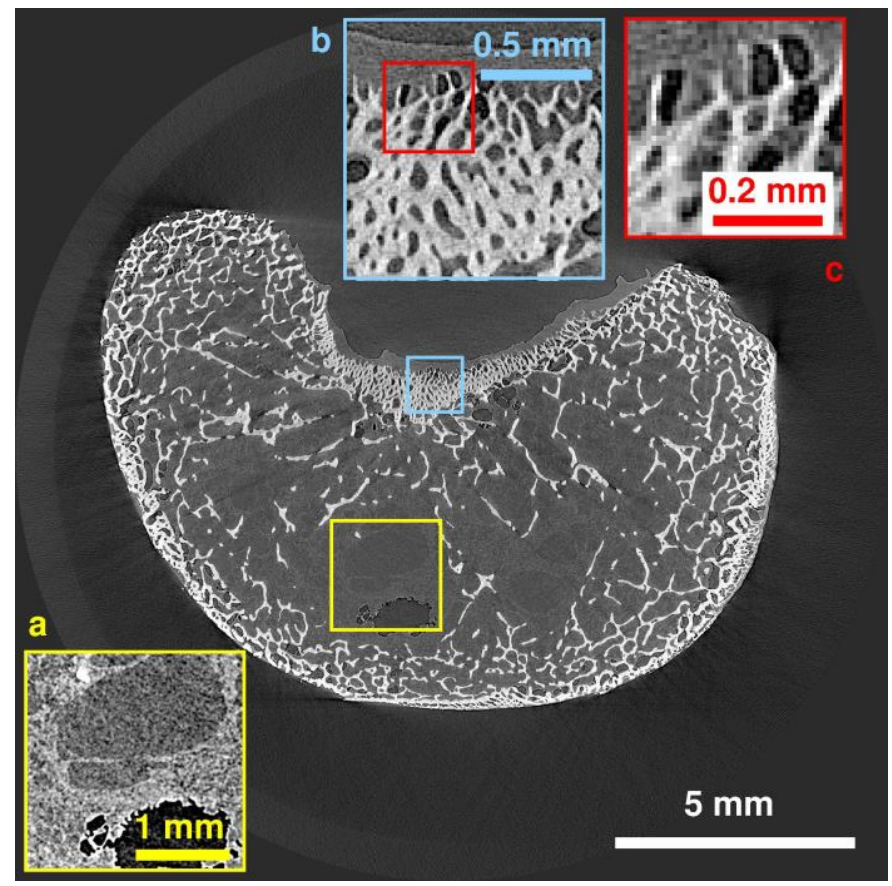

Figure 1. White-beam tomogram of a chicken tibia. Peak energy $20 \mathrm{keV}$, pixel size $9.3 \mu \mathrm{m}$. Details: (a) soft tissue; $(b, c)$ trabecular bone. 1000 projections, exposure time per projection $0.06 \mathrm{~s}$.

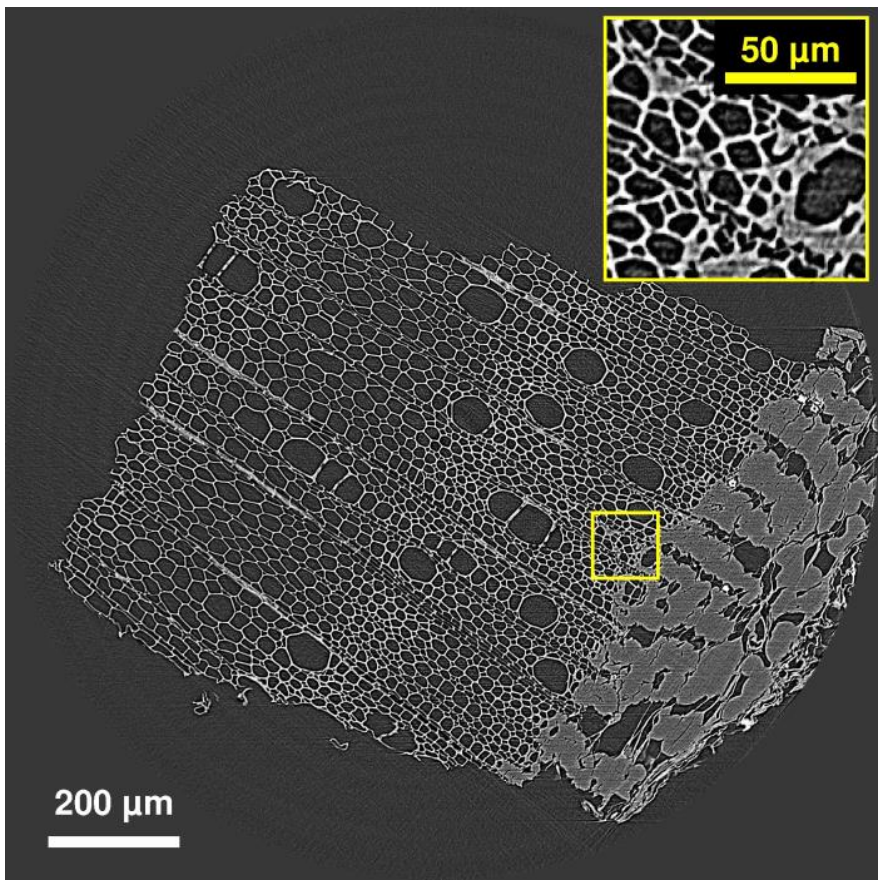

Figure 2. White-beam phase-contrast tomogram of a piece of stem from a hemp plant. Peak energy $10 \mathrm{keV}$, pixel size $0.65 \mu \mathrm{m}$, detector-sample distance $12 \mathrm{~mm} .1500$ projections, scan duration 5 minutes. (Sample: J. Beaugrand, INRA, Nantes.)

The construction of ANATOMIX is largely funded by the French National Research Agency (ANR) through the EQUIPEX investment program, project NanoimagesX, grant no. ANR-11-EQPX-0031. The NanoimagesX consortium consists of 17 partner units from public research and industry. They are acknowledged for their contributions to the functional specifications of the experimental stations.

\section{References:}

[1] T Weitkamp et al, J. Phys. Conf. Series 849 (2017) 012037.

[2] A Somogyi et al, AIP Conf. Proc. 1365 (2011) 57.

[2] M Scheel et al, Microsc. Microanal. (2018), in this volume.

[4] K Desjardins et al, submitted to the $10^{\text {th }}$ International Conference on Mechanical Engineering Design of Synchrotron Radiation Equipment and Instrumentation (MEDSI), 25-29 June 2018, Paris, France.

[5] A King et al, Rev. Sci. Instrum. 87 (2016) 093704. 